\title{
EXAMINING YOUNG NOVICE DRIVER HEADWAY CHOICE IN A SIMULATED CAR FOLLOWING TASK
}

\author{
Eve Mitsopoulos-Rubens, Thomas Triggs, Michael Regan \\ Monash University Accident Research Centre \\ Melbourne, Victoria, Australia \\ E-mail: eve.mitsopoulos@muarc.monash.edu.au
}

\begin{abstract}
Summary: Despite the high incidence of rear-end crashes relative to other crash types among young novice drivers, research examining the car following practices of young novice drivers compared with experienced drivers has been limited. Further, little is understood about the impact of initial following distance - that is, following distance at the commencement of a car following episode - on the final headway adopted. The objective of the current study was to compare systematically the headway choice of a group of 30 young novice drivers with a group of 30 experienced drivers using a car following task that was developed for use in a driving simulator. An important feature of this study was the manipulation of initial following distance. This enabled exploration of how initial headway influences the final headway adopted by each of the novice and experienced groups. The results showed that the young novice drivers in the current study chose to travel at shorter final headways overall than their more experienced counterparts. Furthermore, while initial following distance impacted on the final following distance adopted overall, the novices did not adjust to incremental variations in initial following distance in the same manner as the experienced drivers. The implications of these findings for understanding the mechanisms underlying the headway choices of novice as opposed to experienced drivers are briefly discussed.
\end{abstract}

\section{INTRODUCTION}

The over-representation of young novice drivers in motor vehicle crashes is recognised as a major public health problem. In 2005, in the Australian state of Victoria, 30\% of drivers who died as a result of a motor vehicle crash were aged 18 to 25 years. Astoundingly, this age group constitutes only $14 \%$ of all Victorian licence holders (Transport Accident Commission, 2007). These proportions are not peculiar to Victoria. For example, comparable figures have been found across all 30 OECD countries, which include Australia, France, Sweden, the United Kingdom, and the United States of America (OECD/ECMT, 2006). The mechanisms underlying young novice drivers' high crash involvement are complex, but, in general, they are typically considered to be a reflection of particular skill deficits attributable to inexperience, and volitional risk taking associated with youthfulness and lifestyle (e.g., Drummond, 1989).

Rear-end crashes are among the most common crash types of young novice drivers (Neyens \& Boyle, 2007). Based on 2003 data from the General Estimates System, compiled by the National Highway Traffic Safety Administration, Neyens and Boyle reported that rear-end collisions accounted for $34.9 \%$ of all crashes in which a 16- to 19 -year-old was the driver of the striking vehicle. Moreover, relative to other driver age groups, the largest rear-end crash involvement 
rates are typically found for younger drivers. Drivers aged 24 years and under are also more likely to be in the striking position than in the position of being struck from behind by another vehicle - a pattern which reverses with increasing driver age (Singh, 2003).

Following too closely for the conditions is often cited as an overarching cause of rear-end collisions (e.g., Broughton, Switzer \& Scott, 2007). However, few studies have examined systematically the following distances of young novice drivers relative to experienced drivers. Moreover, results have often been mixed. For example, while some studies indicated shorter headways for young novice drivers relative to older, more experienced drivers (e.g., Evans \& Wasielewski, 1983; Saad, 1996; Taieb-Maimon \& Shinar, 2001), no difference between groups has also been observed (e.g., Colbourn, Brown \& Copeman, 1978; McLaughlin \& Serafin, 2000).

Further, little research (e.g., Eick \& Debus, 2005) concerned with headway preferences in car following has manipulated starting following distance to examine its impact on eventual headway. This is the case even though, in other driving contexts such as speed judgement, the starting condition has been shown to affect the final adopted value (Triggs, 1988). Also, from a practical point of view, a driver might encounter a situation where another vehicle enters into the lane ahead of him/her creating, in effect, an initial following distance that the following driver may elect to modify to some degree by increasing or decreasing his/her speed.

The aim of the current study was to compare systematically the headway choice of a group of young novice drivers with a group of experienced drivers using a car following task developed for use in a driving simulator. An important feature of the study was the manipulation of initial following distance, enabling exploration of how starting distance, ranging from short to long, influences the final headways adopted by novice and experienced drivers. It was expected that the young novice drivers, due to their limited driving experience, would be less adept than their experienced driver counterparts in responding to differences in initial following distance in an adaptive way. This research forms part of a larger project which aims to uncover fundamental differences between novice and experienced drivers' calibration ability - defined as the ability to match the demands of the driving task to one's own driving capabilities (Triggs, 1994).

\section{METHOD}

\section{Experimental Design}

A mixed design with two independent variables was used. The between-subjects factor was driving experience group: novice and experienced. The within-subjects factor was initial following distance. This variable had five levels, ranging from short to long: 12, 23, 34, 45 and 56 metres. At $60 \mathrm{~km} / \mathrm{h}$, the corresponding time values are: 0.7, 1.4, 2.0, 2.7, and 3.4 seconds.

\section{Participants}

Sixty drivers took part in the study. Thirty participants (18 males and 12 females) comprised the novice group and 30 (16 males and 14 females) made up the experienced group. An additional five participants $(7.4 \%$ of the total sample) withdrew from the study due to simulator discomfort. 
A further three participants ( $4.4 \%$ of the total sample), for various reasons, did not provide full data sets and as such their data were not included in the final analyses.

Participants in the novice group ranged in age from 18.1 to 21.7 years (Mean 19.3 years). The minimum licensing age in Victoria, Australia, is 18 years. Participants in the experienced group were aged between 30.4 and 51.3 years (Mean 41.9 years). All novice group participants held a current Victorian Probationary car driver's licence and had held that licence for no more than six months (Mean 3.1 months). Newly licenced drivers in Victoria, Australia, hold a Probationary licence for three years before graduating to a full licence. Participants in the experienced group were all holders of a full car driver's licence, and had been licenced for between 12.4 and 33.3 years (Mean 23.3 years).

\section{Apparatus}

The driving simulator at the Monash University Accident Research Centre (MUARC) in Melbourne, Victoria, Australia, was used in the current study. It consists of a full-size Holden Calais VX sedan with typical interior features. The vehicle is surrounded by two projection screens: one to the front of the vehicle and the other to the rear. The front screen provides a field of view of approximately 180 degrees horizontally and 40 degrees vertically. The rear screen, which was not used in this study, provides a field of view of about 60 degrees horizontally and 40 degrees vertically. Four projectors, three front facing and one rear facing (not used), convey images of pre-programmed traffic scenes onto the screens. The displayed images are updated at a rate of $30 \mathrm{~Hz}$. The simulator vehicle is mounted on a motion platform, providing the driver with some sensation of road feel. A quadraphonic sound system is utilised to provide the driver with realistic traffic sounds, which include engine noise and horn blasts.

The experimenter runs and monitors simulations from the control room, which is located adjacent to the simulator room. A two-way communication system between the control room and the simulator vehicle enables the experimenter and participant to communicate as required. Video monitors located in the control room and connected to cameras mounted inside the vehicle allow the experimenter to monitor participants for signs of discomfort and also the status of the simulations while in progress.

\section{Procedure and Simulator Trials}

Participation involved two sessions conducted on an individual basis and held one to two weeks apart. Each session involved participants completing several activities (e.g., simulator familiarisation drives) in addition to the main activities, which, in the first session comprised the "Autopilot" trials, and in the second session the "Manual" trials. The focus of the current paper is on participants' performance in the Manual trials and, in particular, on their performance in the car following events. Accordingly, only the Manual trials and the car following events are discussed in detail here. Detailed information regarding the Autopilot trials and the other events under study, specifically gap acceptance, can be found in Mitsopoulos, Triggs and Regan (2005, 2006). 
All car following events occurred on a straight section of two-lane residential road. The speed limit was $60 \mathrm{~km} / \mathrm{h}$ and there was some occasional oncoming traffic. Overtaking was not permitted at any time.

Each car following event occurred in two phases. The first phase commenced when a vehicle positioned on the side of the road merged into the simulator vehicle's lane, ahead of the simulator vehicle. Programmed to track at $100 \%$ of the simulator vehicle's speed, the lead vehicle travelled ahead of the simulator vehicle for 250 metres at a previously programmed initial following distance. Participants were instructed to maintain a speed of $60 \mathrm{~km} / \mathrm{h}$ during this time. A single short horn sound signalled the commencement of the second phase of the car following event. During this second phase, the lead vehicle was no longer coupled to the simulator vehicle's speed, and was programmed to travel at $60 \mathrm{~km} / \mathrm{h}$. Once the horn sounded, participants were aware that their task at that point was to modify their speed (i.e., increase, decrease or maintain it) in order for them to reach their preferred headway - that is, a following distance that participants felt was safe for them. While maintaining their preferred following distance, participants were required to return to $60 \mathrm{~km} / \mathrm{h}$ and to continue travelling at that speed until they heard the horn sound twice. At this time, the lead vehicle accelerated and drove into the distance signalling the end of the car following event. This second phase of the car following event took place over a distance of 400 metres.

There were 12 Manual trials in total. Participants first completed two trials for practice. The initial following distance in one practice trial was 18 metres $(1.1$ seconds at $60 \mathrm{~km} / \mathrm{h})$ and in the second it was 50 metres $(3.0$ seconds at $60 \mathrm{~km} / \mathrm{h})$. Presentation of the experimental Manual trials followed. Each initial following distance was presented twice across the ten experimental trials, which were administered to each participant in one of four pseudo-random presentation orders.

\section{RESULTS}

The main dependent variable was mean final following distance or headway. Unless otherwise specified, following distance or headway was defined in the current study as the distance in metres from the rear of the lead vehicle to the front of the simulator (i.e., the driver's) vehicle.

Final headway was said to have been reached during phase two of the car following event once the lead vehicle had stabilised at $60 \mathrm{~km} / \mathrm{h}$ and once the simulator vehicle had returned to within 1 $\mathrm{m} / \mathrm{s}$ of $60 \mathrm{~km} / \mathrm{h}$ subsequent to any gross modifications in speed. These adjustments had occurred by 315 metres into phase two for all participants, on average, in each of the driving experience groups and each of the car following events. Thus, for any given car following event and participant, final following distance was calculated by averaging across all following distance measurements (taken at five metre intervals) in the range beginning with the latest distance during phase two of the car following event where the final headway was reached (315 metres) and ending with the final distance of the car following event (400 metres).

Two questions were of interest here: firstly, is there an effect of driving experience group on final following distance overall; and secondly, is any effect of driving experience group on final following distance contingent on initial following distance? Accordingly, a driving experience group by initial following distance analysis of variance was calculated on the data derived for 
final following distance. The final data, screened for both normality and outliers and averaged across participants, are shown in Figure 1 for each of the novice and experienced groups as a function of initial following distance condition.

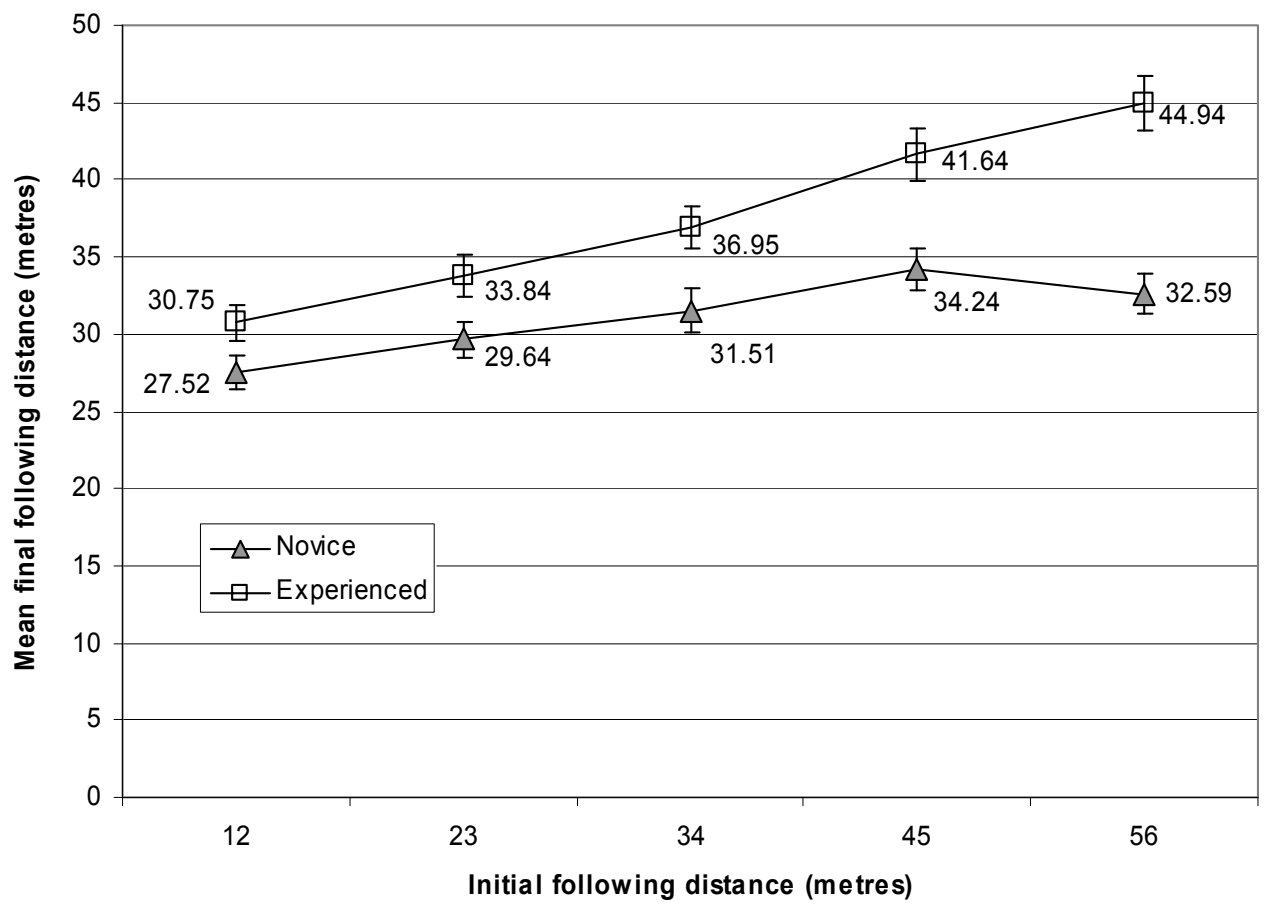

Figure 1. Mean final following distance (metres) for each of the novice and experienced groups as a function of initial following distance condition

The main point to note from Figure 1 is that the mean final following distance is shorter for the novice group than for the experienced group at each of the levels of the initial following distance variable under investigation and, in particular, at the largest initial following distance levels. In line with these observations, a significant main effect of driving experience group was found $(\mathrm{F}(1,116)=14.98, \mathrm{p}=0.00)$, with the novice group adopting a significantly shorter mean final headway overall (31.10 metres) than the drivers in the experienced group (37.62 metres). There was also a significant main effect of initial following distance $(F(3,383)=50.98, p=0.00)$, with shorter initial following distance values resulting in shorter final following distances than the longer initial following distance levels. Importantly, there was a significant interaction between driving experience group and initial following distance $(\mathrm{F}(3,383)=10.01, \mathrm{p}=0.00)$. An analysis of trend for each of the novice and experienced groups, revealed a significant linear component $(F(1,59)=91.18, p=0.00)$ for the experienced group, and significant linear $(F(1,57)=26.41$, $\mathrm{p}=0.00)$ and cubic $(\mathrm{F}(1,57)=4.28, \mathrm{p}=0.04)$ components for the novice group. The quadratic component for the novice group just failed to reach significance $(F(1,57)=3.82, p=0.06)$. In essence, while the experienced drivers showed a consistent increase in their mean final headway with each increment in initial following distance, the novice drivers did not demonstrate this pattern to the same extent. Indeed, at the larger initial following distance conditions, the novice participants appeared to have reached an upper bound in mean final headway. 


\section{CONCLUSIONS}

The aim of the current research was to explore the headway choices of young novice drivers relative to experienced drivers, and to determine whether these choices are affected by initial following distance. The young novice drivers in the current study chose to travel at shorter mean final headways than their more experienced counterparts. Furthermore, while initial following distance impacted on the final following distance adopted overall, consistent with expectations, the novice drivers did not adjust to incremental variations in initial following distance in the same way as the experienced drivers. This was particularly pronounced at the larger initial following distances. One possible explanation for this pattern of results is that, when faced with intermediate to large following distances (i.e., 34, 45 and 56 metres), the novices practised a less skilled "rule-based" approach (Rasmussen, 1983) in electing their preferred following distance. (Recall that, at $60 \mathrm{~km} / \mathrm{h}$, a following distance of 34 metres corresponds to a time headway of 2.0 seconds. In several jurisdictions, 2.0 seconds is considered to be a safe time headway.) Whereas, at the shortest following distances (i.e., 12 and 23 metres), the decision was much easier - that is, to increase following distance to the extent possible in the time available.

The general findings of this study help to corroborate the relatively higher involvement of young drivers in rear-end crashes. They are also consistent with previous research that has shown less conservative following behaviour among novice than experienced drivers. However, while some researchers have attributed shorter following distances among young drivers to an elevated willingness to take risks (e.g., Evans \& Wasielewski, 1983), others have discussed the role of higher order cognitive mechanisms in differentiating the car following practices of novice and experienced drivers (e.g., Saad, 1996). While a role for deliberate risk taking in headway choice cannot be ruled out entirely, the current results add support to the line of thinking that relatively shorter following distances among novice drivers may indeed be a manifestation of less well developed higher order cognitive strategies. This experiment demonstrated a reduced tendency for the novice drivers, relative to the experienced drivers, to respond to differences in initial following distance in an adaptive way. To the knowledge of the authors, this issue has not been explored previously and, as such, warrants further attention.

\section{ACKNOWLEDGMENTS}

The research reported in this paper forms part of the first author's PhD program. Support provided under the Australian Postgraduate Award and the Victorian Government Information and Communication Technologies Scholarship schemes is gratefully acknowledged, as is support for experimental costs provided through the MUARC Doctoral Student Research Fund. The authors also wish to thank Jason Rubens and Ashley Verdoorn for their programming assistance.

\section{REFERENCES}

Broughton, K.L.M., Switzer, F., \& Scott, D. (2007). Car following decisions under three visibility conditions and two speeds tested with a driving simulator. Accident Analysis and Prevention, 39, 106-116.

Colbourn, C.J., Brown, I.D., \& Copeman, A.K. (1978). Drivers' judgments of safe distances in vehicle following. Human Factors, 20, 1-11. 
Drummond, A.E. (1989). An overview of novice driver performance issues: A literature review (Report No. 9). Melbourne, Australia: Monash University Accident Research Centre.

Eick, E., \& Debus, G. (2005). Adaptation effects in an automated car-following scenario. In G. Underwood (Ed.), Traffic and transport psychology: Theory and application. Amsterdam: Elsevier, 243-255.

Evans, L., \& Wasielewski, P. (1983). Risky driving related to driver and vehicle characteristics. Accident Analysis and Prevention, 15, 121-136.

McLaughlin, S., \& Serafin, C. (2000). On-road investigation of driver following and deceleration on surface streets. Proceedings of the IEA/FES 2000 Congress (Vol. 3). San Diego, USA, 294-297.

Mitsopoulos, E., Triggs, T., \& Regan, M. (2005). Investigating the calibration ability of young novice drivers relative to experience drivers: Preliminary findings from a driving simulator study. Proceedings of the 2005 Australasian Road Safety Research, Policing and Education Conference. Wellington, New Zealand.

Mitsopoulos, E., Triggs, T., \& Regan, M. (2006). Examining novice driver calibration through novel use of a driving simulator. Proceedings of SimTect2006. Melbourne, Australia.

Neyens, D.M., \& Boyle, L.N. (2007). The effect of distractions on the crash types of teenage drivers. Accident Analysis and Prevention, 39, 206-212.

OECD/ECMT. (2006). Young drivers: The road to safety (Summary document). Available at http://www.cemt.org/JTRC/WorkingGroups/YoungDrivers/YoungDriversSummary.pdf

Rasmussen, J. (1983). Skills, rules, and knowledge: Signals, signs, and symbols, and other distinctions in human performance models. IEEE Transactions on Systems, Man, and Cybernetics, SMC-13, 257-266.

Saad, F. (1996). Driver strategies in car-following situations. In A.G. Gale, I.D. Brown, C.M. Haslegrave \& S.P. Taylor (Eds.), Vision in vehicles - V. Amsterdam: Elsevier, 61-70.

Singh, S. (2003). Driver attributes and rear-end crash involvement propensity (Report No. DOT HS 809 540). Washington, DC: National Highway Traffic Safety Administration.

Taieb-Maimon, M., \& Shinar, D. (2001). Minimum and comfortable driving headways: Reality versus perception. Human Factors, 43, 159-172.

Transport Accident Commission. (2007). Young driver statistics. Available at http://www.tacsafety.com.au/jsp/content/NavigationController.do?areaID=12

Triggs, T.J. (1988). Speed estimation. In G.A. Peters \& B.J. Peters (Eds.), Automotive engineering and litigation (Vol. 2). New York: Garland Law Publishing, 569-598.

Triggs, T.J. (1994). Human performance and driving: The role of simulation in improving young driver safety. Proceedings of the $12^{\text {th }}$ Triennial Congress of the International Ergonomics Association (Vol. 1). Toronto, Canada, 23-26. 